\title{
The molecular basis for adhesion-mediated suppression of reactive oxygen species generation by human neutrophils
}

\author{
Tieming Zhao, ${ }^{1}$ Valerie Benard, ${ }^{2}$ Benjamin P. Bohl, ${ }^{1}$ and Gary M. Bokoch ${ }^{1,3}$ \\ ${ }^{1}$ Department of Immunology, The Scripps Research Institute, La Jolla, California, USA \\ 2Unité Mixte de Recherche 5018, Centre National de la Recherche Scientifique, Toulouse, France \\ ${ }^{3}$ Department of Cell Biology, The Scripps Research Institute, La Jolla, California, USA
}

\begin{abstract}
Human neutrophil adherence to ECMs induces an initial inhibition of stimulated reactive oxygen species (ROS) formation, followed by an enhanced phase of oxidant production. The initial integrinmediated suppression of ROS constitutes a mechanism to prevent inappropriate tissue damage as leukocytes migrate to inflammatory sites. The Rac2 guanosine 5' -triphosphatase (GTPase) is a critical regulatory component of the phagocyte NADPH oxidase. We show that activation of Rac2 is inhibited in adherent neutrophils, correlating with inhibition of ROS formation. Conversely, NADPH oxidase components $\mathrm{p} 47$ and $\mathrm{p} 67$ assemble normally, suggesting a specific action of adhesion on the Rac2 molecular switch. Reconstitution with activated Rac2 restored rapid NADPH oxidase activation kinetics to adherent neutrophils, establishing that inhibition was due to defective Rac2 activity. We provide evidence that integrins inhibit Rac2 activation via a membrane-associated guanine nucleotide exchange factor, likely to be Vav1. Activation of Vav1, but not its upstream activator, Syk, is suppressed by cell adhesion. Vav1 activity is inhibited due to dephosphorylation of the regulatory Tyr174 via enhanced tyrosine phosphatase activity in adherent cells. These studies identify an integrin-mediated pathway in which Vav1 is as a strong candidate for the critical regulatory point in suppression of Rac2 activation and ROS generation during inflammatory responses.
\end{abstract}

J. Clin. Invest. 112:1732-1740 (2003). doi:10.1172/JCI200319108.

\section{Introduction}

Neutrophils and related phagocytic leukocytes play important roles in human host defense against a wide range of infectious agents. These cells ingest invading microorganisms and kill them via the generation of reactive oxygen species (ROS), termed the "respiratory burst." The initial formation of superoxide anion from molecular oxygen occurs through the activity of the NADPH oxidase (1). Superoxide gives rise to hydrogen peroxide spontaneously or via superoxide dismutase and to hypochlorous acid via the primary granule protein myeloperoxidase. However, inappropriate and/or excessive production of ROS will also exacerbate the inflammatory process and cause tissue damage (2). Thus, the "double-edged

Received for publication June 3, 2003, and accepted in revised form September 30, 2003.

Address correspondence to: Gary M. Bokoch, Departments of Immunology and Cell Biology, The Scripps Research Institute, 10550 North Torrey Pines Road, La Jolla, California 92037, USA. Phone: (858) 784-8217; Fax: (858) 784-8218;

E-mail: bokoch@scripps.edu.

Conflict of interest: The authors have declared that no conflict of interest exists.

Nonstandard abbreviations used: reactive oxygen species (ROS); fMetLeuPhe (fMLF); complement component 5a (C5a); guanosine $5^{\prime}$-triphosphate (GTP); guanosine $5^{\prime}$-triphosphatase (GTPase); tetratricopeptide repeat (TPR); guanine nucleotide exchange factor (GEF); glutathione-S-transferase-p21 binding domain (GST-PBD); diphenyliodonium (DPI); immunoblot (IB); guanosine 5'-diphosphate (GDP); GDP dissociation inhibitor (GDI); GTPase-activating protein (GAP); protein tyrosine phosphatase (PTP); proline, glutamate, serine, threonine (PEST). sword" of ROS production needs to be tightly regulated in order to maintain homeostasis in addition to an effective host defense.

The adherence of neutrophils to a variety of ECM proteins (fibronectin, fibrinogen, laminin, and the like) has been shown to result in the initially delayed, but subsequently enhanced, formation of ROS in response to a variety of soluble activators, including fMetLeuPhe (fMLF), GM-CSF, TNF- $\alpha$, complement component $5 \mathrm{a}$ (C5a), and IL-8, for example (3-5). The initial suppression of ROS formation by adhesion is thought to represent a protective mechanism to prevent inappropriate tissue damage as neutrophils travel through tissues to reach the inflammatory site(s), where enhanced ROS formation is desired. This phenomenon of adhesionmediated regulation of the NADPH oxidase of human neutrophils has been well documented; it is independent of secretion or protein synthesis and requires signaling via leukocyte integrins (6-8). Inhibition is observed even after cells have been allowed to preadhere and spread but could be ablated by cytoskeletal disruption with dihydrocytochalasin b (3). The molecular basis for the inhibitory crosstalk between adhesion receptors and chemoattractant and/or cytokine receptors involved in regulating the NADPH oxidase of neutrophils has not been elucidated.

NADPH oxidase is an inducible electron transport system found in a variety of cell types, including leukocytes, that transfers reducing equivalents from NADPH to oxygen, resulting in the production of superoxide anion $(9,10)$. The NADPH oxidase consists of a mem- 
brane-integrated cytochrome $b_{558}$, composed of two subunits (gp91 phox (phagocytic oxidase) and gp22phox), and several cytosolic proteins ( $\mathrm{p}^{4} 7^{\text {phox }}, \mathrm{p} 67^{\mathrm{phox}}, \mathrm{Rac}$ guanosine 5'-triphosphate (GTP); guanosine 5'-triphosphatase (GTPase), and possibly p40 phox). NADPH oxidase exists in a dormant unassembled state in resting cells but, upon stimulation, p47 and p67 translocate to the plasma membrane and form a complex with cytochrome $b$. Rac GTPase is activated and translocates to the membrane independently of the translocation of $\mathrm{p} 47$ and $\mathrm{p} 67$ and is an indispensable component of this active oxidase complex $(11,12)$. GTP-bound Rac interacts directly with p67 via its tetratricopeptide repeat (TPR) motif $(13,14)$ and with cytochrome $b$ via its insert domain (15), and these interactions are crucial for transferring electrons to cytochrome $b$ and molecular oxygen.

In the present study, we have investigated the mechanism of adhesion-induced suppression of human leukocyte NADPH oxidase activity. Our results demonstrate that Rac2 GTPase serves as a common point of convergence for crosstalk between leukocyte adhesion and chemoattractant receptors. Integrins act to suppress Rac2 activation and assembly into the functional membrane NADPH oxidase complex. This inhibitory effect is associated with a decrease in the activation of the Vav1 Rac guanine nucleotide exchange factor (GEF). These findings provide important molecular insights into how $\mathrm{NADPH}$ oxidase may be regulated during the processes of transendothelial migration, chemotaxis, and subsequent activation of neutrophils at inflammatory sites.

\section{Methods}

Preparation of neutrophils. Human blood was collected from The Scripps Research Institute General Clinical Research Center donor pool by venipuncture, and the neutrophils isolated by dextran sedimentation, hypotonic lysis of erythrocytes, and centrifugation through Ficoll-Paque (Amersham-Pharmacia Biotech, Uppsala, Sweden) as described previously (12). The cells were washed with $0.9 \% \mathrm{NaCl}$ and resuspended in KrebsRinger HEPES buffer (118 mM NaCl, $4.8 \mathrm{mM} \mathrm{KCl}, 25$ mM HEPES, $1.2 \mathrm{mM} \mathrm{KH}_{2} \mathrm{PO}_{4}, 1.2 \mathrm{mM} \mathrm{MgSO}_{4}$ ) containing $5.5 \mathrm{mM}$ glucose for experiments in both adherent and suspension conditions.

Measurement of hydrogen peroxide formation. For adherent cells, 12-well tissue culture plates (Becton Dickinson, Franklin Lakes, New Jersey, USA) were coated with $50 \mu \mathrm{g} / \mathrm{ml}$ polylysine, $50 \mu \mathrm{g} / \mathrm{ml}$ human plasma fibronectin, or $20 \mu \mathrm{g} / \mathrm{ml}$ fibrinogen (Sigma-Aldrich, St. Louis, Missouri, USA) overnight at $4{ }^{\circ} \mathrm{C}$, then washed with PBS three times. After blocking with $3 \%$ BSA for 1 hour, the plates were again washed with PBS three times. For those experiments in which cells were pretreated with $200 \mu \mathrm{M}$ of orthovanadate $\left(\mathrm{Na}_{3} \mathrm{VO}_{4}\right)$ or 5 $\mu \mathrm{g} / \mathrm{ml}$ of cytochalasin b (Sigma-Aldrich), the neutrophils were prewarmed at $37^{\circ} \mathrm{C}$ for 10 minutes in the presence of inhibitor or vehicle, then the cells were added to the plates in the presence of $1 \times 10^{-7} \mathrm{M}$ fMLF.
For suspension conditions, cells were added to polypropylene microfuge tubes coated with BSA. The tubes were placed on a horizontal shaker at 200 cycles $/ \mathrm{min}$ at $37^{\circ} \mathrm{C}$. After stimulation with fMLF, neutrophils were then collected at various times and hydrogen peroxide formation determined using the homovanilic acid-based assay (16). In some experiments, neutrophils were transduced with recombinant WT Rac2 and Rac2(V12) mutant GTPases at $10 \mu \mathrm{g} / \mathrm{ml}$ using Bioporter reagent (Gene Therapy Systems, San Diego, California, USA), as described (17).

Rho GTPase pull-down assay and Pak, Vav1, and Syk activity assay. Rac2 activation assays were performed using $0.4-\mathrm{mg}$ cell lysates and $10 \mu \mathrm{g}$ of glutathione-Stransferase-p21 binding domain (GST-PBD), essentially as described (18). Proteins were separated by $12 \%$ SDS-PAGE, transferred to PVDF membrane, and blotted with specific Rac2 R786 antibody (19). Pak activity was measured using in-gel kinase assay with the p47phox peptide, amino acid 297-331, as a substrate (20). Pak (1 and 2) immunoprecipitations were performed using anti-Pak antibody 2124/3 (21). In experiments determining tyrosine phosphorylation of Vav1 and Syk, $0.4 \mathrm{mg}$ cell lysates were incubated with rabbit anti-Vav1 or anti-pTyr174 Vav antibody, or rabbit anti-Syk antibody (Santa Cruz Biotechnology, Santa Cruz, California, USA). Immune complexes were captured by protein A Sepharose and immunoblotted using antibody against phosphotyrosine (4G10; Upstate Biotechnology, Lake Placid, New York, USA). Fifty micrograms of cell lysates were immunoblotted with rabbit anti-Vav1 or anti-Syk antibody to assess total Vav1 and Syk levels in lysates. Specific tyrosine phosphorylation of Vav1 was verified using a second Vav1-specific antiserum kindly provided by Victor Tybulewicz (National Institute for Medical Research, London, United Kingdom).

Membrane translocation assay. Membranes and cytosol of human neutrophils were separated by ultracentrifugation as described previously (12). Briefly, neutrophils were disrupted by two 7 -second pulses of sonication using a microtip probe sonicator (Heat Systems, Farmingdale, New York, USA) at low amplification. Unbroken cells and nuclei were removed by low-speed centrifugation, and the resulting supernatant loaded onto $6.7 \mathrm{ml}$ of $15 \%$ sucrose layered over $4 \mathrm{ml}$ of $34 \%$ sucrose. After centrifugation for 40 minutes at $4^{\circ} \mathrm{C}$ and 195,000 $g$, cytosol was removed from the top of the gradient and the light membrane fraction collected from the interface of the $15 \%$ and $34 \%$ sucrose solution. The cytosol and membranes were then subjected to SDS-PAGE, followed by immunoblotting with specific antisera to assess translocation of NADPH oxidase components to the membrane $(19,22)$.

Cell-free NADPH oxidase activity assay. Production of $\mathrm{O}_{2}{ }^{-}$was measured in a cell-free system as previously described (23). Ten micrograms of membranes and 70 $\mu \mathrm{g}$ of cytosol were mixed together with $0.1 \mathrm{mM}$ cytochrome $c$ in a total volume of $150 \mu$ l. Reactions 


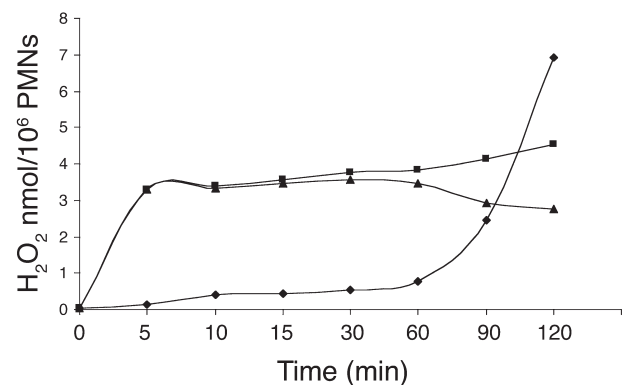

Figure 1

Adhesion to fibronectin inhibits the oxidative response of chemoattractant-stimulated neutrophils. $\mathrm{H}_{2} \mathrm{O}_{2}$ production was measured as described in Methods at various times after stimulation with $\mathrm{fMLF}$ $\left(1 \times 10^{-7} \mathrm{M}\right)$ in nonadherent neutrophils (squares) or neutrophils plated on immobilized fibronectin $(50 \mu \mathrm{g} / \mathrm{ml})$ (diamonds) or polyL-lysine $(50 \mu \mathrm{g} / \mathrm{ml})$ (triangles). Results shown are representative of more than 25 independent experiments using cells from different donors. PMN, polymorphonuclear leukocyte.

were initiated by adding SDS to a final concentration of $90 \mu \mathrm{M}$. Initial rates of reduction of cytochrome $c$ at $550 \mathrm{~nm}$ were measured using Softmax software (Molecular Devices, Sunnyvale, California, USA).

\section{Results}

Cell adhesion suppresses NADPH oxidase activation. $\mathrm{H}_{2} \mathrm{O}_{2}$ production was used as a measure of ROS formation via NADPH oxidase in freshly prepared human neutrophils immobilized on fibronectin in the presence of $1 \times 10^{-7} \mathrm{M}$ fMLF as activator (16). Cells in suspension or attached on a positively charged polylysine surface were used as controls, as neither of these conditions engage cellular integrins. Consistent with the original observations of Nathan (3), the oxidase response of chemoattractant-stimulated adherent human neutrophils was divided into two phases: a dramatically delayed or inhibited phase lasting from 30 to 60 minutes, followed by a phase of enhanced oxidant generation lasting several hours (Figure 1). $\mathrm{H}_{2} \mathrm{O}_{2}$ formation reached a maximum of approximately $2-4 \mathrm{nmol} / 10^{6}$ neutrophils in suspension cells within 5 minutes, whereas adherent cells reached a maximum of approximately $20-30 \mathrm{nmol} / 10^{6}$ neutrophils after 3-4 hours. Cells in suspension or attached to polylysine did not exhibit a delayed oxidase response (Figure 1), verifying previous observations that this effect is not simply induced by cell attachment, but rather by integrin-mediated signaling. Allowing neutrophils to adhere and spread prior to stimulation with $\mathrm{AMLF}$ did not eliminate the inhibitory effect on $\mathrm{H}_{2} \mathrm{O}_{2}$ formation, and suppression was observed when cellular $\beta_{1}$ or $\beta_{2}$ integrins were activated with specific antibodies (not shown), consistent with the need for $\beta$ integrin-dependent signals $(5-7,24,25)$.
Activation of Rac2 and the effector Pak correlates with the kinetics of the oxidative response during cell adhesion. Since Rac is a common signaling component in both adherence and NADPH oxidase activation, we examined the regulation of this GTPase during the inhibitory phase of ROS production induced in adherent neutrophils. Activities of Rac2 GTPase and its effector p21-activated kinase (Pak) were determined in adherent versus suspension neutrophils using an affinity-based Rac GTPase activation assay. This assay is based upon the specific binding of Rac2GTP to the p21-binding domain of Pak1 (18). Activity of the Rac effectors, Paks 1 and 2, were determined by in-gel kinase assay (20). Rac2 and Pak (Paks 1 and 2) activation induced by fMLF paralleled oxidant production kinetics in adherent versus suspension cells (Figure 2), whereas no activation was observed in unstimulated (i.e., no fMLF) adherent or suspension cells. At the later stages in the adherent cells when formation of ROS again became evident, we noted that Rac2 activity progressively increased and was subsequently maintained at levels equivalent to or greater than the levels obtained at any time points in suspension cells (not shown). These data suggest that integrin signaling regulates both NADPH oxidase activity and Rac GTPase in a parallel manner. Treatment of cells with diphenyliodonium (DPI), an effective inhibitor of NADPH oxidase (26), at concentrations that fully blocked ROS formation did not interfere with the activation of Rac2 observed at later times, indicating that Rac2 activation was not the result of oxidant formation (Figure 2).

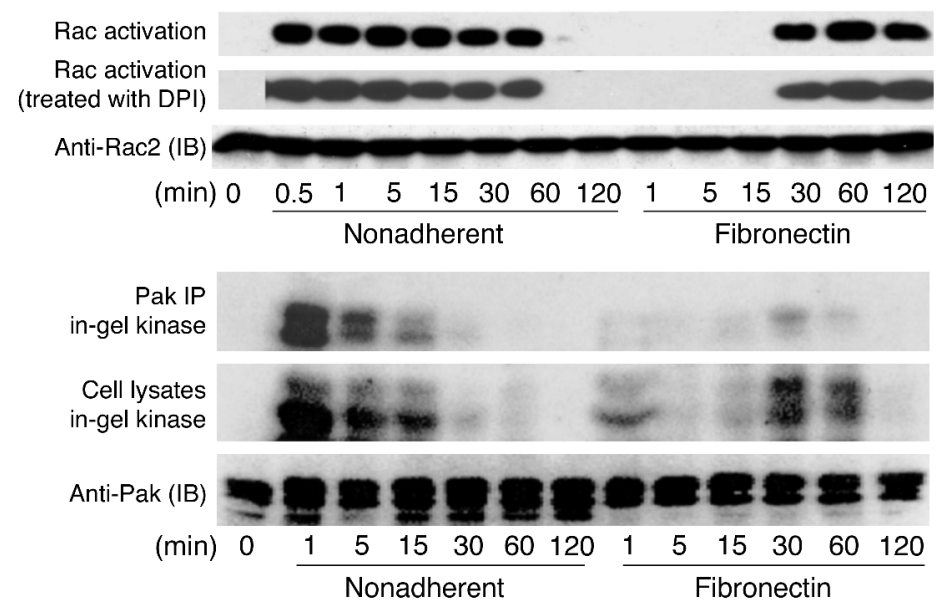

Figure 2

Adhesion of neutrophils to fibronectin leads to delayed Rac2 and Pak activation in response to $\mathrm{fMLF}$ stimulation. Neutrophils plated on fibronectin (50 $\mu \mathrm{g} / \mathrm{ml}$ ) or in nonadherent conditions were treated with $1 \times 10^{-7} \mathrm{M}$ fMLF. After the times indicated, Rac2 activity in the absence or presence of $10 \mu \mathrm{M} \mathrm{DPI}$ was determined, as in Methods. Total Rac2 levels (anti-Rac2 IB) did not change with time under each condition. Pak1 and Pak2 activities were analyzed by ingel kinase assays of immunoprecipitated (IP) Pak or directly in cell lysates. IBs in the bottom panel show that Pak ( 1 and 2 ) levels did not change with time under each condition. The results shown are representative of four similar experiments obtained with cells derived from different donors. 


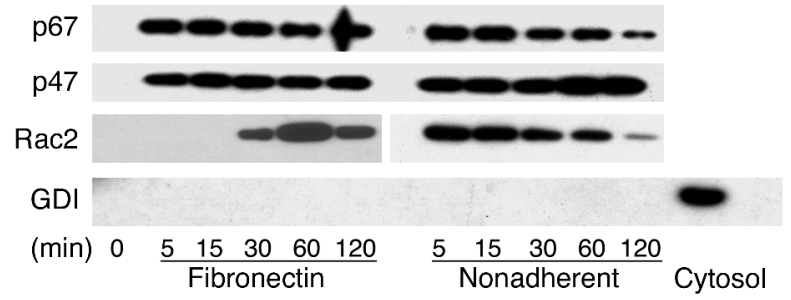

Figure 3

Adhesion of neutrophils to immobilized fibronectin leads to delayed membrane translocation of Rac2, but not 447 and p67. Neutrophils plated on immobilized fibronectin or in nonadherent conditions were treated with $1 \times 10^{-7} \mathrm{M} \mathrm{MLF}$. After the times indicated, the cells were fractionated as in Methods, and the presence of Rac2, p47, p67, and RhoGDI on the cell membrane were analyzed. The last lane of the GDI panel represents $10 \mu \mathrm{g}$ of cytosol as a RhoGDI antibody control. Results shown are representative of three independent experiments using cells from different donors.

Translocation of Rac2, but not $p 47$ and $p 67$, is regulated by cell adhesion. Translocation and membrane assembly of the cytosolic NADPH oxidase components (p47, p67, and Rac2) to the cell membrane is critical to formation of the active oxidase. We assessed whether membrane translocation of Rac2, p47, and p67 was normal in adherent versus suspension neutrophils (Figure 3). Interestingly, we observed that translocation of p47 and p67 to the membrane was not affected by cell adhesion as compared with suspension cells. In marked contrast, Rac2 translocation was delayed, and this delay correlated with the delay in Rac2 activation in adherent cells. These data indicate that adhesion signals suppress NADPH oxidase through regulation of Rac activity/function but not via inhibition of the assembly of the other cytosolic components, p47 and p67.

Constitutively active Rac2 is sufficient to restore normal NADPH oxidase response kinetics in adherent cells. In order to establish the inhibition of Rac2 function as the critical regulatory point in adherence-mediated NADPH oxidase suppression, we introduced either wild-type or the constitutively active Rac2(V12) mutant into intact neutrophils. The suppression of ROS formation observed in untreated adherent leukocytes was completely reversed by reconstitution with Rac2(V12), but not by WT Rac2 (Figure 4a). These data strongly indicate that the action of integrin signaling to suppress NADPH oxidase activation occurs primarily via regulation of Rac2 GTPase function and that the function of the other oxidase components is normal. The fact that normal activation kinetics were restored by constitutively activated Rac2(V12) but not by WT Rac2 also indicated that there was no intrinsic defect in Rac2 in the adherent cells but rather that the ability of the GTPase to become activated or to maintain the active GTP state was being suppressed.

Adhesion suppresses Rac2 activation. In order to determine whether adhesion modulates the activity of cytosolic or membrane-associated components involved in NADPH oxidase and/or Rac2 activation, cytosol and membrane fractions were prepared from suspended and adherent neutrophils at early ( 0 minutes and 5 minutes, respectively) times after stimulation with fMLF, and the ability to support NADPH oxidase activity in a cell-free assay was determined (27). Consistent with the intact cell studies, the mixture of membranes and cytosol prepared from adherent cells during the inhibited phase is defective in oxidant formation, whereas the mixture of membranes and cytosol from suspended cells supports a normal level of NADPH oxidase activity (Figure $4 \mathrm{~b}$ ).

A series of mix-and-match experiments in which membranes and cytosol from each condition were mixed and assayed was performed (27). We observed that the mixture of membranes derived from adherent cells and cytosol derived from suspension cells is defective in NADPH oxidase activity but noted that the mixture of membranes from resting cells and the cytosol from adherent cells is normal. These data indicate that adhesion induces a defect at the level of a plasma membrane component. This defect in function of a membrane component can be overcome by providing con-

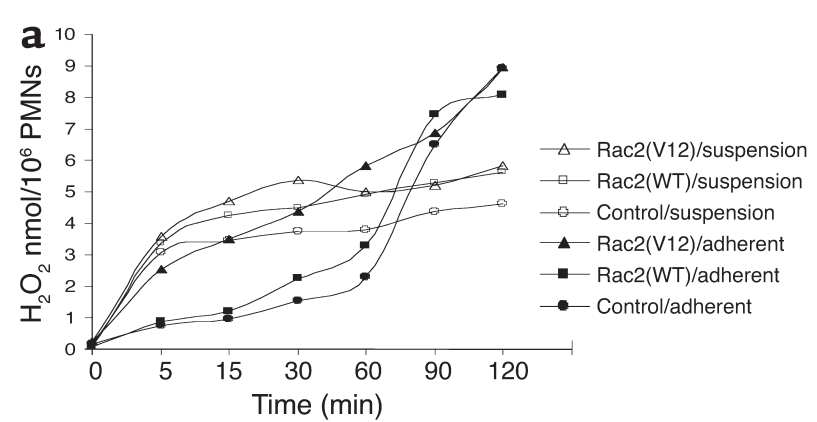

b

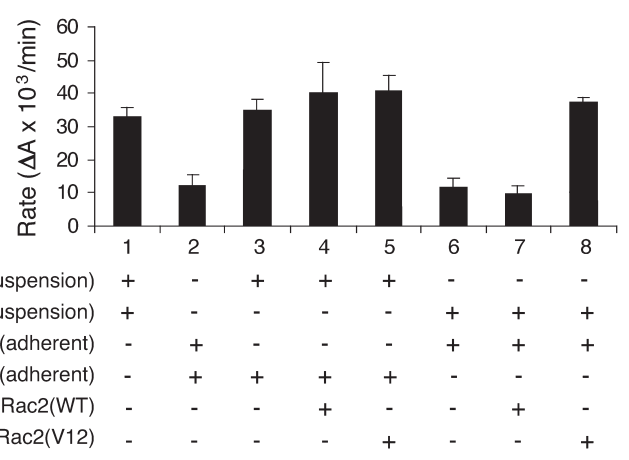

\section{Figure 4}

Constitutively active Rac2 restores adhesion-mediated inhibition of $\mathrm{NADPH}$ oxidase activity in neutrophils and a reconstituted cell-free system. (a) Nontreated neutrophils, neutrophils expressing WT Rac2, or cells expressing constitutively active Rac2(V12), as described in Methods, were tested for $\mathrm{H}_{2} \mathrm{O}_{2}$ production in response to $1 \times 10^{-7} \mathrm{M}$ fMLF under suspension and adherent conditions. Results shown are representative of three independent experiments using cells from different donors. (b) Human neutrophil membrane and cytosol fractions were prepared from suspended or adherent cells at early ( 0 minutes and 5 minutes, respectively) times after stimulation with $1 \times 10^{-7}$ $\mathrm{M} \mathrm{fMLF}$, as described in Methods. Superoxide anion formation was determined with various mixtures of membranes and cytosols, as indicated. Results shown are the mean \pm SD of three independent experiments. $\Delta \mathrm{A}$, change in absorbance at $550 \mathrm{~nm}$. 

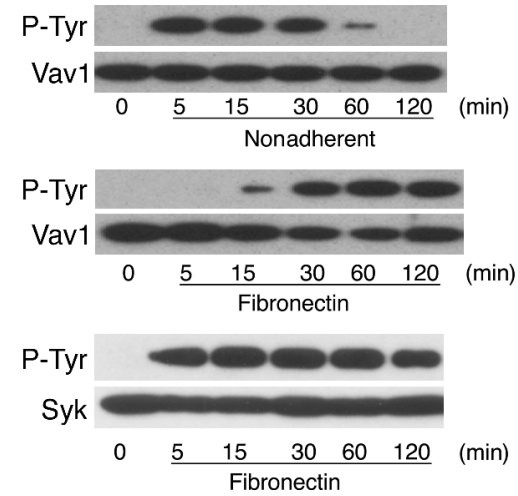

\section{Figure 5}

Adhesion of neutrophils to fibronectin leads to delayed Vav1 activation but early Syk activation in response to fMLF stimulation. Neutrophils plated on fibronectin $(50 \mu \mathrm{g} / \mathrm{ml})$ or in nonadherent conditions were treated with $1 \times 10^{-7} \mathrm{M} \mathrm{fMLF}$. After the times indicated, Vav1 and Syk tyrosine phosphorylation (P-Tyr) was determined, as described in Methods. Total Vav1 and Syk levels (anti-Vav1 and antiSyk IB) were constant with time under each condition. The results shown are representative of three similar experiments obtained with cells derived from different donors.

stitutively active Rac2(V12), but not WT Rac2 (Figure $4 \mathrm{~b})$. These observations strongly suggest that adhesion signaling inhibits the ability of a membrane-associated Rac GEF to catalyze guanine nucleotide exchange on Rac2, resulting in disruption of the process of Rac2 activation. Alternatively, the activity of a membraneassociated Rac2 GAP might be stimulated by adhesion (see Discussion). The ability of cytosol from the inhibited phase of adherent neutrophils to support normal NADPH oxidase activation again indicates there was no intrinsic defect in endogenous Rac2, nor in its ability to be activated in response to the membrane-localized GEF from suspended cells.

Activation of the Vav1 Rac GEF is deficient in adherent neutrophils. There are two Rac GEFs that have been implicated in regulation of human neutrophil NADPH oxidase function: Vav1 (28) and P-Rex1 (29). Although P-Rex1 appears to be regulated solely by $G$ proteincoupled receptors, Vav1 has been shown to be regulated downstream of integrin engagement through the Syk-mediated tyrosine phosphorylation of the critical Tyr174 residue in Vav1 (30-32). We therefore examined Vav1 activation in adherent versus suspension neutrophils by assessing the tyrosine phosphorylation of the Tyr 174 activation site (Figure 5). Of particular interest, we observed that activation of Vav1 in AMLFstimulated neutrophils adherent to fibronectin was suppressed as compared with suspension cells. No Vav1 activation was observed in the absence of fMLF in either suspended or adherent conditions. The delay in Vav1 Tyr174 phosphorylation in adherent cells correlated exactly with the onset of Rac 2 activation and oxidant production when analyzed in the same experiment. Similar activation kinetics and suppression were observed when Western blotting was performed with anti-pTyr174 Vav antibody, followed by Vav1 immunoblot (IB) (not shown). In contrast, activity of Syk, the immediate upstream regulator of Vav1 Tyr174 phosphorylation, was not suppressed in adherent cells and Syk was activated at early time points (Figure 5). An integrin-mediated negative regulatory pathway thus exists that prevents Vav1 activation subsequent to the activation of Syk in adherent neutrophils.

Inhibition of Rac2 and Vav1 is a common feature of adhesion-mediated inhibitory NADPH oxidase regulation. We wanted to determine if inhibition of Rac2 activity and Vav1 activation was a common component of inhibitory signaling by integrins to ROS production. We therefore examined whether inhibition of ROS formation observed by activation of $\beta 2$ integrins also occurred through suppression of Rac2 and Vav1. Cells plated on a fibrinogen matrix, which acts via $\beta 2$ integrins, exhibited an initially delayed oxidative response to $\mathrm{MLF}$, similar to that shown with the fibronectin matrix ( $\beta 1$ integrins) in Figure 1 (data not shown). As demonstrated in Figure 6, we observed that during the inhibitory phase induced by adhesion on fibrinogen, both Rac2 and Vav1 activity were suppressed to an extent comparable to that observed on a fibronectin matrix.

We next examined whether the inhibition of Rac2 and Vav1 activation was a specific effect resulting from the use of $\mathrm{MLF}$ as an activating stimulus. The complement $\mathrm{C} 5 \mathrm{a}$ is a chemoattractant that acts through distinct $G$ protein-coupled receptors to stimulate NADPH oxidase activity. Cells stimulated with C5a exhibited ROS formation kinetics similar to those seen with fMLF when applied to neutrophils adherent on fibronectin or fibrinogen versus suspension cells (data not shown). Oxidant formation was again correlated with activation of Rac2 and Vav1 in the C5a-treated cells (Figure 6). Thus, this pathway

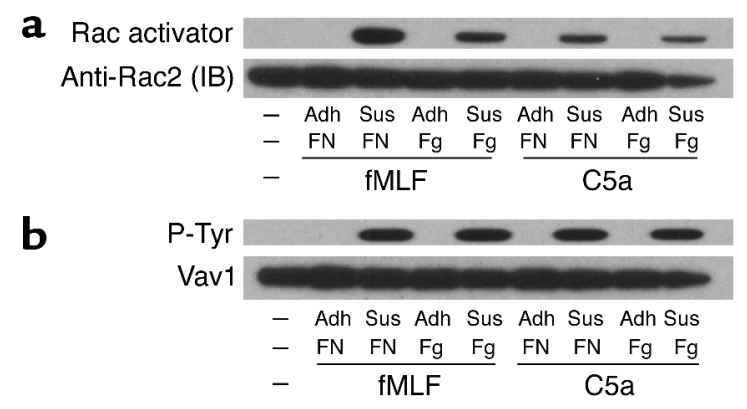

Figure 6

Adhesion (Adh) of neutrophils to fibronectin (FN) or fibrinogen (Fg) leads to delayed Rac2 and Vav1 activation in response to fMLF or C5a stimulation. Neutrophils plated on fibronectin (50 $\mu \mathrm{g} / \mathrm{ml})$, fibrinogen $(20 \mu \mathrm{g} / \mathrm{ml})$, or in nonadherent (Sus) conditions were treated with $1 \times 10^{-7} \mathrm{M}$ fMLF or $10 \mathrm{nM} \mathrm{C} 5 \mathrm{a}$. After 5 minutes, Rac2 (a) and Vav1 (b) activities were determined, as described in Methods. Total Rac2 and Vav1 levels (anti-Rac2 and anti-Vav1 IB) were constant under each condition. The results shown are representative of two similar experiments obtained with cells derived from different donors. 
a

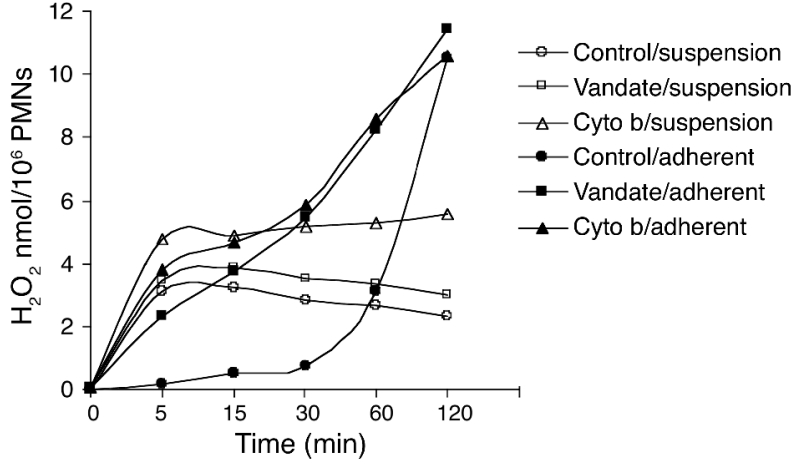

b

Rac activation

Anti-Rac2 (IB)

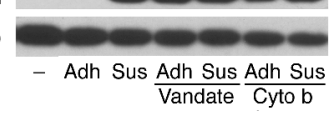

c

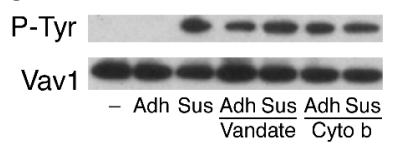

Figure 7

Tyrosine phosphatase inhibition and cytoskeletal disruption restore adhesioninhibited NADPH oxidase activity and Rac2 and Vav1 activation. Neutrophils treated with vanadate or cytochalasin b (cyto b) as in described in Methods were tested for $\mathrm{H}_{2} \mathrm{O}_{2}$ production in response to $1 \times 10^{-7} \mathrm{M} \mathrm{AMLF}$ under suspension and adherent conditions (a). Rac2 (b) and Vav1 (c) activities were determined after treatment with $\mathrm{MLF}$ for 5 minutes. Results shown are representative of two independent experiments using cells from different donors.

appears to account for the suppression of NADPH oxidase function induced by two distinct types of $\beta$ integrin and in response to cell activation by two separate inflammatory mediators.

Integrins act through tyrosine phosphatase(s) to suppress Vav1 activity. Previous studies have shown that disruption of the actin cytoskeleton by treatment of neutrophils with agents such as cytochalasins ablates the integrin-mediated inhibitory phase of ROS formation (3). We have confirmed this observation and show that this reversal of the inhibitory phase of ROS formation is correlated with a restoration of both Rac2 and Vav1 activation (Figure $7 \mathrm{~b}$ ). The integrin-mediated regulatory pathway thus appears to be dependent on the presence of an intact actin cytoskeleton. We have not ruled out other mechanisms, however.

In order to begin to address the identity of the integrinregulated component that decreases phosphorylation of Tyr174 in Vav1, we examined the incorporation of phosphotyrosine overall into neutrophil proteins. Lysates prepared from either adherent or nonadherent neutrophils at $t=0$ or 5 minutes were analyzed by phosphotyrosine blot (phosphotyrosine antibody 4G10) after SDS-PAGE. We observed a general decrease in the incorporation of phosphotyrosine into multiple protein bands on the blot in the adherent cells when compared with suspension cells (data not shown). This overall decrease in phosphotyrosine suggested that, rather than having an inhibitory effect on specific kinases, adhesion was inducing enhanced activity of tyrosine phosphatase(s).

We therefore examined the effect of the tyrosine phosphatase inhibitor, orthovanadate, on adhesionmediated ROS suppression. As shown in Figure 7a, treatment of adherent neutrophils with orthovanadate caused a dramatic enhancement of $\mathrm{H}_{2} \mathrm{O}_{2}$ formation at early times. In contrast, there was no significant increase in the rate of $\mathrm{H}_{2} \mathrm{O}_{2}$ formation at late times when the system normally exhibited an oxidative burst. Similarly, orthovanadate did not significantly enhance $\mathrm{H}_{2} \mathrm{O}_{2}$ formation in suspension cells at any time. These results supported the hypothesis that adhesion enhanced the activity of protein tyrosine phosphatase(s) that regulate the phosphorylation state of Vav1. Consistent with this, we observed that orthovanadate completely reversed the inhibition of Rac2 activation and Vav1 Tyr174 phosphorylation induced by adhesion while not affecting Rac2 or Vav1 activation in suspension cells (Figure 7, b and c).

\section{Discussion}

Evidence that engagement of adhesion receptors can induce a suppression of ROS generation was first provided by Fehr et al. (33) and Nathan (3). Nathan showed that adhesion of neutrophils to surfaces coated with ECM proteins (fibronectin, fibrinogen, laminin, and the like) resulted in a dramatically delayed oxidative response to soluble cytokine stimuli (fMLF, TNF- $\alpha$, and so on). Lasting from 30 to 90 minutes, this delay was not decreased by preincubation with either soluble activator or ECM, nor by allowing the cells to preadhere and spread. Furthermore, the NADPH oxidase could be rapidly activated with phorbol esters, such as phorbol 12-myristate 13-acetate, indicating that adherence specifically affected chemoattractant and cytokine signaling pathways. These studies suggested that a common component of integrin signaling is involved in NADPH oxidase regulation by adhesion. However, the molecular basis for the inhibitory crosstalk between adhesion receptors and chemoattractant receptors involved in downregulating the oxidative responses of human neutrophils remained unknown.

There is substantial evidence that both chemoattractant receptor-mediated regulation of leukocyte NADPH oxidase and integrin-mediated adhesive signaling involve small GTPases of the Rho family, particularly Rac $(34,35)$. Upon stimulation of neutrophils, Rac2 is dissociated from cytosolic complexes with guanosine $5^{\prime}$-diphosphate dissociation inhibitor (GDP dissociation inhibitor, GDI) by an unknown mechanism that may involve a membrane protein and/or the formation of specific lipids (36). At this point, GTP is exchanged for GDP on Rac2 through the action of an unidentified membrane-associated GEF (22). Active Rac2 consequently assembles as part of the functional NADPH oxidase complex at the plasma membrane (19). This process is dissociable from the translocation of p67 and p47 to the membrane $(11,12)$, and membrane-associated Rac2 is necessary for significant superoxide production. Within the assembled NADPH 
oxidase membrane complex, the GTP-bound Rac interacts directly with p67 via its TPR repeat motif $(13,14)$ and with cytochrome $b$ through its insert helix (15). These interactions are crucial for transferring electrons from NADPH to cytochrome $b$ and molecular oxygen. When ECM ligands are engaged by integrins, both Rac and Cdc42 GTPases are activated by undefined mechanisms and, along with their target kinases, initiate cellular signaling cascades (37). Cell spreading occurs after initial attachment of cells to immobilized ECM and is characterized by membrane ruffling, correlating with the activation of $\operatorname{Rac}$ and $\operatorname{Cdc} 42(38,39)$. Spreading is blocked by dominant negative forms of Rac and Cdc42. In contrast, Rho activity decreases during the initial spreading phase, followed by an increase as spreading reaches completion and stress fibers became prominent (40).

We show in the present study that Rac GTPase serves as a common point of convergence for integrin and chemoattractant receptor crosstalk. First, we verified prior observations that adhesion signals induce suppression of NADPH oxidase activation lasting 30-90 minutes (Figure 1). This integrin-mediated suppression was observed in cells plated on either fibronectin ( $\beta 1$ integrins) or fibrinogen ( $\beta 2$ integrins) matrices, and with either AMLF or C5a as activating stimuli (Figure 6). Using an affinity-based GST-fusion protein pull-down assay, we measured Rac2 activation during the process of adhesion-regulated oxidant formation and found a very close correlation between these two events (Figure 2). Again, we found that inhibition of Rac2 activity occurred with either fibronectin or fibrinogen matrix and with both $\mathrm{PMLF}$ and C5a as activating agents (Figure 6). Studies on NADPH oxidase assembly showed that adhesion signals resulted in delayed Rac2 translocation to the cell membrane but that the other cytosolic NADPH oxidase components, p47 and p67, assembled normally at the membrane (Figure 3 ). These data suggested that adhesion signals regulate NADPH oxidase by specifically controlling the activation and translocation of the Rac2 regulatory component. This hypothesis was verified by demonstrating that reconstitution of intact neutrophils and a cell-free oxidase system with constitutively active Rac2 protein reversed the suppressive effects of adhesion on NADPH oxidase activity (Figure 4a). The inability of WT Rac2 to restore activity indicates that the functionality and/or availability of endogenous Rac2 in the adherent cells (e.g., due to inability to be released from GDI complexes) is not a limiting factor. Active Rac is thus necessary and sufficient to reverse the effects of adhesion, indicating that Rac GTPase serves as a critical regulatory point in suppression of oxidant formation by integrins. Indeed, our data indicate that Rac2 serves as a direct activator of NADPH oxidase activity in adherent cells. The fact that membrane translocation of the cytosolic oxidase components, p47 and p67, occurred in the absence of Rac2 activation and that full NADPH oxidase activity could be restored by the reconstitution of the preassembled system with Rac2, rules out any required translocating function of Rac2 under these conditions. The current observations are consistent with the earlier finding that Rac2 plays an important direct regulatory role in electron transport reactions catalyzed by cytochrome $b(15)$.

This time-dependent, integrin-mediated inhibition of Rac activation contrasts with the rapid stimulatory effects of integrins on Rac activation observed in fibroblasts $(38,39)$. Neutrophil $\beta$ integrins thus appear to engage a unique inhibitory signaling pathway to modulate Rac activity associated with the process of ROS generation. Of interest, an integrinmediated inhibitory pathway that suppresses Rac activation in the context of motility and metastasis has been reported. Wnt-induced-secreted-protein-1 inhibits Rac activation in an $\alpha_{v} \beta_{5}$-dependent manner in H460 lung cancer cells (41). Transfection of the cells with a constitutively active RacG12V mutant restored cell motility and invasiveness. While this article was in review, Dib et al. (42) also reported that $\beta$ integrin engagement inhibited Rac1 and Rac2 activation in human neutrophils stimulated by the soluble mediator TNF- $\alpha$.

We were able to localize the defect in Rac2 activation to a plasma membrane-associated component (Figure $4 b)$. Our data do not support a model in which Rac might be sequestered by membrane integrins, as we did not observe Rac2 associated with the cell membrane during the adhesion-induced inhibitory phase. It is also unlikely that adhesion acted to mask membrane binding sites for Rac, since we could readily restore NADPH oxidase activity by the addition of Rac2 $(12 \mathrm{~V})$ to either intact cells or the cell-free assay system. Indeed, the latter observation strongly indicates that adhesion induces either an inability to activate Rac2 or an enhanced capability to stimulate Rac2 deactivation via a membrane-localized component. Based upon previous observations, this is most likely to represent suppression of a membrane-associated Rac GEF (22). Dib et al. reported that adhesion stimulated the activity of unidentified Rac GTPase-activating proteins (GAPs) in TNF- $\alpha$-stimulated neutrophils (42). The effect on Rac GAP activity they detected was modest and seems unlikely to account for the total loss of Rac activation observed. In our cell-free studies (Figure 4B), we observed that NADPH oxidase activity was suppressed even in the presence of the nonhydrolyzable guanine nucleotide GTP $\gamma$, making it unlikely to be due to an enhanced GAP activity.

Indeed, correlating with the inhibition of Rac2 activation, we observed that activation of the membraneassociated Rac GEF, Vav1, was inhibited in adherent neutrophils. Vav1 phosphorylation on the critical Tyr174 site that is indicative of Vav1 GEF activity was observed only at later times when Rac2 activity and oxidant formation also became evident (Figure 5). This inhibition of Vav1 tyrosine phosphorylation was also common to inhibition observed in cells on a fibronectin versus fibrinogen matrix and in cells activated 
by either $\mathrm{AMLF}$ or $\mathrm{C} 5 \mathrm{a}$ (Figure 6). In contrast, Vav1 was activated in response to PMLF at early times in suspension cells, again correlating exactly with the onset of Rac2 activity and oxidant formation. We used two Vav1-specific antibodies (see Methods) to verify that we were observing activity of this hematopoietic cell-specific GEF. Vav1 activation via the phosphorylation of Tyr174 has been shown to be dependent upon the upstream tyrosine kinase Syk in hematopoeitic cells $(30,31)$. Of particular interest, we observed that Syk activation was rapid in fMLF-stimulated adherent neutrophils, becoming highly active at early time points at which Vav1 activation was not evident. This suggests either that Syk no longer has access to the Vav1 substrate or raises the possibility of the activation by adhesion of one or more tyrosine phosphatase(s) capable of dephosphorylating Vav1 at Tyr174. The kinase Pyk2 has also been reported to bind and regulate Vav1 activation in certain leukocytes (43). We observed that Pyk2 activation was rapid in adherent neutrophils and that its activity did not correlate with the onset of Vav1 activity (data not shown).

The effects of adhesion on NADPH oxidase activation, Rac2 activity, and Vav1 tyrosine phosphorylation were all reversed (Figure 7) in the presence of the general tyrosine phosphatase inhibitor sodium orthovanadate (44). In conjunction with an observed generalized decrease in tyrosine-phosphorylated proteins in adherent cells, this is consistent with the adhesionmediated activation of one or more tyrosine phosphatases that can regulate the activation state of Vav1. In various cells, integrins have been shown to stimulate the activity of a number of tyrosine phosphatases, protein tyrosine phosphatase-1B (PTP-1B) (45) and PTP-proline, glutamate, serine, threonine (PTP-PEST) (46). Our preliminary studies suggest that the inhibitory pathway of $\beta$ integrins in human neutrophils may involve more than one tyrosine phosphatase ( $T$. Zhao and G.M. Bokoch, unpublished observations). We cannot, however, rule out at this time other indirect effects of vanadate, including possible activation of kinases.

We have described here a previously uncharacterized integrin-mediated inhibitory pathway that suppresses signaling resulting from the activation of upstream tyrosine kinases (i.e., Syk) coupled to Vav1 and, consequently, results in defective activation of the crucial NADPH oxidase regulatory component, Rac2 GTPase. Our data place Vav1 as a likely critical regulator of Rac2 activation coupled to NADPH oxidase activation in adherent neutrophils. Consistent with these results, it has recently been shown that leukocytes from Vav1 null mice exhibit dramatic defects in ROS formation induced by PMLF (M. Dinauer, personal communication). These observations clearly suggest that Vav1 is an important link between $G$ protein-coupled chemoattractant receptors and Rac2 activation coupled to NADPH oxidase activation.

To participate effectively in host defense, neutrophils must respond to chemotactic factors $(N$-formylated bacterial peptides, C5a, leukotriene B4, IL-8, and the like) and cytokines (e.g., TNF- $\alpha$ ), leave the bloodstream, and migrate across an endothelial cell monolayer into the tissues to contain and eliminate the infectious agents. Stimulation of chemoattractant receptors in nonadherent neutrophils results in a very rapid (within minutes) activation of the cells, leading to degranulation and to generation of ROS via the NADPH oxidase. This rapid and continuous ROS formation is unlikely to occur physiologically, as it would be teleologically undesirable for leukocytes to secrete toxic oxygen radicals as they migrate through the tissues to inflammatory sites. The prolonged lag period in the oxidative response observed in leukocytes adherent to surfaces coated with ECMs is presumed to correspond to the in situ situation in which the cells are migrating through the tissue to inflammatory sites. After the cells reach these sites, the leukocytes then respond and generate microbicidal oxidants. Adhesion signaling events mediated by cell surface integrin receptors are thus key steps in the processes of transendothelial migration (47), and, according to our present study, regulation of $\mathrm{NADPH}$ oxidase responsiveness through the Rac2 GTPase serves as a crucial point of regulation by integrin signaling. Indeed, we note that after the initial inhibited phase, the subsequent activity of Rac2 appears to be both higher and prolonged in adherent neutrophils, suggesting that enhanced ROS formation at this stage could be due to increased Rac GTPase activity. This will be investigated in future studies. The identification of Vav1 as the major point of inhibitory crosstalk between adhesion receptors and cytokine receptors during NADPH oxidase regulation provides significant new insight into regulation of the inflammatory process and may lead to an improved understanding of the pathology and treatment of inflammation-related disorders.

\section{Acknowledgments}

This study received ethical approval from The Scripps Research Institute review board. This work was supported by grants HL48008 and GM44428 from the National Institutes of Health (to G.M. Bokoch) and a fellowship from the Arthritis Foundation (to T. Zhao).

1. Clark, R.A. 1990. The human neutrophil respiratory burst oxidase. J. Infect. Dis. 161:1140-1147.

2. Smith, J.A. 1994. Neutrophils, host defense, and inflammation: a double-edged sword. J. Leukoc. Biol. 56:672-686.

3. Nathan, C.F. 1987. Neutrophil activation on biological surfaces. Massive secretion of hydrogen peroxide in response to products of macrophages and lymphocytes. J. Clin. Invest. 80:1550-1560.

4. Nathan, C.F. 1989. Respiratory burst in adherent human neutrophils: triggering by colony-stimulating factors CSF-GM and CSF-G. Blood. 73:301-306.

5. Nathan, C., et al. 1989. Cytokine-induced respiratory burst of human neutrophils: dependence on extracellular matrix proteins and CD11/CD18 integrins. J. Cell Biol. 109:1341-1349.

6. Shappell, S.B., et al. 1990. Mac-1 (CD11b/CD18) mediates adherencedependent hydrogen peroxide production by human and canine neutrophils. J. Immunol. 144:2702-2711.

7. Liles, W.C., Ledbetter, J.A., Waltersdorph, A.W., and Klebanoff, S.J. 1995 Cross-linking of CD18 primes human neutrophils for activation of the respiratory burst in response to specific stimuli: implications for adhesion-dependent physiological responses in neutrophils. J. Leukoc. Biol. 58:690-697. 
8. Dusi, S., Della Bianca, V., Donini, M., Nadalini, K.A., and Rossi, F. 1996 Mechanisms of stimulation of the respiratory burst by TNF in nonadherent neutrophils: its independence of lipidic transmembrane signaling and dependence on protein tyrosine phosphorylation and cytoskeleton. J. Immunol. 157:4615-4623.

9. Babior, B.M., Lambeth, J.D., and Nauseef, W. 2002. The neutrophil NADPH oxidase. Arch. Biochem. Biophys. 397:342-344.

10. Vignais, P.V. 2002. The superoxide-generating NADPH oxidase: struc tural aspects and activation mechanism. Cell. Mol. Life Sci. 59:1428-1459.

11. Dorseuil, O., Quinn, M.T., and Bokoch, G.M. 1995. Dissociation of Rac translocation from $\mathrm{p} 47 \mathrm{phox} / \mathrm{p} 67$ phox movements in human neutrophils by tyrosine kinase inhibitors. J. Leukoc. Biol. 58:108-113.

12. Heyworth, P.G., Bohl, B.P., Bokoch, G.M., and Curnutte, J.T. 1994. Rac translocates independently of the neutrophil NADPH oxidase components $\mathrm{p} 47 \mathrm{phox}$ and $\mathrm{p} 67 \mathrm{phox}$. Evidence for its interaction with flavocytochrome b558. J. Biol. Chem. 269:30749-30752.

13. Koga, H., et al. 1999. Tetratricopeptide repeat (TPR) motifs of p67(phox) participate in interaction with the small GTPase Rac and activation of the phagocyte NADPH oxidase. J. Biol. Chem. 274:25051-25060.

14. Lapouge, K., et al. 2000. Structure of the TPR domain of p67phox in complex with Rac.GTP. Mol. Cell. 6:899-907.

15. Diebold, B.A., and Bokoch, G.M. 2001. Molecular basis for Rac2 regulation of phagocyte NADPH oxidase. Nat. Immunol. 2:211-215.

16. Ruch, W., Cooper, P.H., and Baggiolini, M. 1983. Assay of $\mathrm{H} 2 \mathrm{O} 2$ production by macrophages and neutrophils with homovanillic acid and horse-radish peroxidase. J. Immunol. Methods. 63:347-357.

17. Gardiner, E.M., et al. 2002. Spatial and temporal analysis of Rac activation during live neutrophil chemotaxis. Curr. Biol. 12:2029-2034.

18. Benard, V., Bohl, B.P., and Bokoch, G.M. 1999. Characterization of rac and cdc42 activation in chemoattractant-stimulated human neutrophils using a novel assay for active GTPases. J. Biol. Chem. 274:13198-13204.

19. Quinn, M.T., Evans, T., Loetterle, L.R., Jesaitis, A.J., and Bokoch, G.M. 1993. Translocation of Rac correlates with NADPH oxidase activation. Evidence for equimolar translocation of oxidase components. J. Biol. Chem. 268:20983-20987.

20. Ding, J., Knaus, U.G., Lian, J.P., Bokoch, G.M., and Badwey, J.A. 1996. The renaturable 69- and 63-kDa protein kinases that undergo rapid activation in chemoattractant-stimulated guinea pig neutrophils are p21-activated kinases. J. Biol. Chem. 271:24869-24873.

21. Dharmawardhane, S., Sanders, L.C., Martin, S.S., Daniels, R.H., and Bokoch, G.M. 1997. Localization of p21-activated kinase 1 (PAK1) to pinocytic vesicles and cortical actin structures in stimulated cells. J. Cell Biol. 138:1265-1278.

22. Bokoch, G.M., Bohl, B.P., and Chuang, T.H. 1994. Guanine nucleotide exchange regulates membrane translocation of Rac/Rho GTP-binding proteins. J. Biol. Chem. 269:31674-31679.

23. Heyworth, P.G., et al. 1993. Requirement for posttranslational processing of Rac GTP-binding proteins for activation of human neutrophil NADPH oxidase. Mol. Biol. Cell. 4:261-269.

24. Berton, G., Laudanna, C., Sorio, C., and Rossi, F. 1992. Generation of signals activating neutrophil functions by leukocyte integrins: LFA-1 and gp150/95, but not CR3, are able to stimulate the respiratory burst of human neutrophils. J. Cell Biol. 116:1007-1017.

25. Lowell, C.A., Fumagalli, L., and Berton, G. 1996. Deficiency of Src family kinases p59/61hck and p58c-fgr results in defective adhesion-dependent neutrophil functions. J. Cell Biol. 133:895-910.

26. Cross, A.R. 1990. Inhibitors of the leukocyte superoxide generating oxidase: mechanisms of action and methods for their elucidation. Free Radic. Biol. Med. 8:71-93.

27. Bokoch, G.M., and Prossnitz, V. 1992. Isoprenoid metabolism is required for stimulation of the respiratory burst oxidase of HL-60 cells. J. Clin. Invest. 89:402-408.

28. Price, M.O., Atkinson, S.J., Knaus, U.G., and Dinauer, M.C. 2002. Rac activation induces NADPH oxidase activity in transgenic COSphox cells, and the level of superoxide production is exchange factor-dependent. J. Biol. Chem. 277:19220-19228.

29. Welch, H.C., et al. 2002. P-Rex1, a PtdIns(3,4,5)P3- and Gbetagammaregulated guanine-nucleotide exchange factor for Rac. Cell. 108:809-821.

30. Miranti, C.K., Leng, L., Maschberger, P., Brugge, J.S., and Shattil, S.J. 1998. Identification of a novel integrin signaling pathway involving the kinase Syk and the guanine nucleotide exchange factor Vav1. Curr. Biol. 8:1289-1299.

31. Moores, S.L., et al. 2000. Vav family proteins couple to diverse cell surface receptors. Mol. Cell. Biol. 20:6364-6373.

32. Aghazadeh, B., Lowry, W.E., Huang, X.Y., and Rosen, M.K. 2000. Structural basis for relief of autoinhibition of the Dbl homology domain of proto-oncogene Vav by tyrosine phosphorylation. Cell. 102:625-633.

33. Fehr, J., Moser, R., Leppert, D., and Groscurth, P. 1985. Antiadhesive properties of biological surfaces are protective against stimulated granulocytes. J. Clin. Invest. 76:535-542.

34. Bokoch, G.M., and Diebold, B.A. 2002. Current molecular models for NADPH oxidase regulation by Rac GTPase. Blood. 100:2692-2696.

35. Schwartz, M.A., and Shattil, S.J. 2000. Signaling networks linking integrins and rho family GTPases. Trends Biochem. Sci. 25:388-391.

36. Chuang, T.H., Bohl, B.P., and Bokoch, G.M. 1993. Biologically active lipids are regulators of Rac.GDI complexation. J. Biol. Chem. 268:26206-26211.

37. Yamada, K.M., and Miyamoto, S. 1995. Integrin transmembrane signaling and cytoskeletal control. Curr. Opin. Cell Biol. 7:681-689.

38. Clark, E.A., King, W.G., Brugge, J.S., Symons, M., and Hynes, R.O. 1998. Integrin-mediated signals regulated by members of the rho family of GTPases. J. Cell Biol. 142:573-586.

39. Price, L.S., Leng, J., Schwartz, M.A., and Bokoch, G.M. 1998. Activation of Rac and Cdc42 by integrins mediates cell spreading. Mol. Biol. Cell. 9:1863-1871.

40. Ren, X.D., Kiosses, W.B., and Schwartz, M.A. 1999. Regulation of the small GTP-binding protein Rho by cell adhesion and the cytoskeleton. EMBO J. 18:578-585.

41. Soon, L.L., et al. 2003. Overexpression of WISP-1 down-regulated motility and invasion of lung cancer cells through inhibition of Rac activation. J. Biol. Chem. 278:11465-11470.

42. Dib, K., et al. 2003. Down-regulation of Rac activity during beta 2 integrinmediated adhesion of human neutrophils. J. Biol. Chem. 278:24181-24188.

43. Gismondi, A., et al. 2003. Proline-rich tyrosine kinase 2 and rac activation by chemokine and integrin receptors controls NK cell transendothelial migration. J. Immunol. 170:3065-3073.

44. Huyer, G., et al. 1997. Mechanism of inhibition of protein-tyrosine phosphatases by vanadate and pervanadate. J. Biol. Chem. 272:843-851.

45. Arregui, C.O., Balsamo, J., and Lilien, J. 1998. Impaired integrin-mediated adhesion and signaling in fibroblasts expressing a dominant-negative mutant PTP1B. J. Cell Biol. 143:861-873.

46. Sastry, S.K., Lyons, P.D., Schaller, M.D., and Burridge, K. 2002. PTP-PEST controls motility through regulation of Rac1. J. Cell Sci. 115:4305-4316.

47. Albelda, S.M., Smith, C.W., and Ward, P.A. 1994. Adhesion molecules and inflammatory injury. FASEB J. 8:504-512. 